\title{
High-frequency repetitive transcranial magnetic stimulation for treating moderate traumatic brain injury in rats: A pilot study
}

\author{
XIA LU $^{1 *}$, XINJIE BAO $^{1 *}$, JIANTAO LI $^{1 *}$, GUANGHAO ZHANG $^{2}$, JIAN GUAN $^{1}$, \\ YUNZHOU GAO ${ }^{3}$, PEILIN WU ${ }^{4}$, ZHAOHUI ZHU ${ }^{4}$, XIAOLIN HUO ${ }^{2}$ and RENZHI WANG ${ }^{1}$ \\ ${ }^{1}$ Department of Neurosurgery, Peking Union Medical College Hospital, Chinese Academy of Medical Sciences and Peking \\ Union Medical College, Beijing 100005; ${ }^{2}$ Laboratory of Bioelectromagnetics, Institute of Electrical Engineering, \\ Chinese Academy of Sciences, Beijing 100190; Departments of ${ }^{3}$ Histology and Anatomy and \\ ${ }^{4}$ Nuclear Medicine, Peking Union Medical College Hospital, \\ Chinese Academy of Medical Sciences and Peking Union Medical College, Beijing 100005, P.R. China
}

Received February 3, 2016; Accepted February 3, 2017

DOI: $10.3892 /$ etm.2017.4283

\begin{abstract}
Transcranial magnetic stimulation (TMS) is a method of noninvasive brain stimulation that causes neuromodulation by activating neurons or changing excitability in a certain brain area. Considering the known effects of TMS and the pathophysiology of traumatic brain injury (TBI), TMS was proposed to have potential for treating this condition. Moderate TBI was induced in adult male Sprague Dawley rats using Feeney's weight-dropping method. Injured rats were divided into a TMS group and a control group. Repetitive TMS (rTMS) was administered to rats in the TMS group from post-TBI day 2. At post-TBI days 7, 14 and 28, three or four of the rats were sacrificed, and harvested brains were embedded in paraffin and sectioned. Sections were then treated with hematoxylin and eosin and immunohistochemical staining. Three rats from each group underwent fludeoxyglucose F 18 micro-positron emission tomography scanning on post-TBI day 2 and 13. The unexpected mortality rate after injury was lower in the TMS group than in the control group. The modified neurological severity score of the TMS group was lower compared with the control group at post-TBI day 14. According to the results of hematoxylin eosin staining, relative cerebral parenchyma loss was lower at post-TBI day 28 in the TMS group compared with the control group. However, the aforementioned differences were not found to be statistically significant. There was also no significant difference in glucose metabolism between the
\end{abstract}

Correspondence to: Dr Renzhi Wang, Department of Neurosurgery, Peking Union Medical College Hospital, Chinese Academy of Medical Sciences and Peking Union Medical College, 1 Shuaifuyuan, Beijing 100005, P.R. China

E-mail: wangrz@126.com

*Contributed equally

Key words: traumatic brain injury, transcranial magnetic stimulation, neuromodulation, neural stem cell, neurogenesis two groups. According to immunohistochemical staining, the TMS group showed a significantly higher level of proliferation (indicated by bromodeoxyuridine) in the subventricular zone, as compared with the control group $(\mathrm{P}<0.05)$. A significantly higher rate of neuron survival at day $28(\mathrm{P}<0.05$; indicated by $\mathrm{NeuN}$ ) and a significantly reduced rate of apoptosis at days 7 and $14(\mathrm{P}<0.05$; indicated by caspase-3) were observed in the perilesional zone of the TMS group, as compared with the control group. The current findings suggest that high-frequency rTMS may promote neurogenesis and provide a basis for further studies in this area.

\section{Introduction}

Traumatic brain injury (TBI) is common in all populations, regardless of age, background or general health condition (1). In the United States, 1.7 million people suffer from TBI every year (2). TBI has a high mortality rate and frequently leads to disability in survivors (3). According to a large study conducted in 2004, traffic accidents caused $60.9 \%$ of TBI cases in eastern China (4).

Based on the known pathophysiology of TBI, neuromodulation technology has been inferred to have potential for treating TBI. Noninvasive brain stimulation (NBS) exhibits particular potential because it can change neuronal excitability or cause neuronal discharges without any invasive procedures $(5,6)$. Previous results have supported the use of NBS techniques for enhancing motor skills and cognitive function in healthy subjects, and as therapeutic methods for patients with neurological and psychiatric disorders (7).

Transcranial magnetic stimulation (TMS) is an NBS technique based on electromagnetic stimulation that was first introduced in 1985 (8). Repetitive TMS (rTMS) can modulate cortical excitability, with low-frequency $(\leq 1 \mathrm{~Hz})$ stimulation causing a decrease in excitability (9) and high-frequency $(\geq 5 \mathrm{~Hz}$ ) stimulation resulting in increased excitability (10). These techniques are known as inhibitory and excitatory rTMS, respectively. In the course of TBI pathophysiology, primary injury in the early phase is caused by a decreased blood flow to the perilesional zone (1). Previous studies have 
suggested that excitatory rTMS over the cortex can increase regional blood flow, while inhibitory rTMS can lead to a decrease (11-14). In the later phase of TBI pathophysiology, synaptic reorganization can be either beneficial or detrimental, also known as adaptive or maladaptive plasticity (5). Excitatory rTMS can promote neuroplasticity, and when combined with task performance training, has the potential to promote adaptive plasticity (5). Additionally, excitatory rTMS has been demonstrated to persistently increase levels of the neuroprotective brain-derived neurotrophic factor (BDNF) in rats (15). Therefore, it was considered worthwhile to investigate the potential of rTMS for treating TBI.

In the current study, it was hypothesized that excitatory rTMS could improve outcomes in TBI. This was investigated by applying high-frequency rTMS to rats with induced TBI, and subsequently observing parameters reflecting tissue protection and neural regeneration.

\section{Materials and methods}

Animal models. All animal procedures conformed to the guidelines issued by the Committee on Animal Research of Peking Union Medical College Hospital (Beijing, China) and were approved by the Institutional Ethics Committee. A total of 38 adult (age, 10 weeks) male Sprague Dawley ${ }^{\circledR}$ rats (weight, $227.5 \pm 9.6 \mathrm{~g}$ ) were purchased from Beijing HFK Bioscience Co., Ltd. (Beijing, China). Rats were housed in cages at $15-25^{\circ} \mathrm{C}$, in a room with a 12/12-h light/dark cycle. Food and water were available ad libitum. TBI was induced using Feeney's weight-dropping impact method, as previously described (16). The footplate on which the dropped weight impacted was directly attached to the dura and was $4.6 \times 4.0 \mathrm{~mm}$ in area. Before the impact, each rat was anesthetized with an intraperitoneal injection of $10 \%$ chloral hydrate $(400 \mathrm{mg} / \mathrm{kg}$; Sinopharm Chemical Reagent Co., Ltd, Shanghai, China), then a 5-mm diameter bone window was created, with the center located $2.5 \mathrm{~mm}$ lateral to the central suture and $1.5 \mathrm{~mm}$ behind the bregma. The force of impact to the right hemispherical cortex was $20 \mathrm{~g}$ x $25 \mathrm{~cm}$. According to Gao et al (17), this method should cause moderate TBI. All craniotomies and impacts were performed by an experienced researcher within a two-week period.

Behavioral tests and grouping. Evaluation of the modified neurological severity score (mNSS) was performed at post-TBI days 1,7,14 and 28. The mNSS is a composite of motor, sensory, balance and reflex tests. Neurological function is graded on a scale of 0-18 (normal score $=0$; maximal deficit score $=18$ ). One point is gained for each specific abnormal behavior or lack of a tested reflex; thus, a higher score indicates more severe injury. This test is suitable for evaluating long-term neurological outcomes after unilateral brain injury (18). Rats were pre-trained for the tests used to determine mNSS for three days before induction of TBI. Evaluation was accomplished by an experienced researcher who was blinded to the grouping. TBI rats were hierarchically divided based on mNSS scores and rats of with the same score were subsequently randomly distributed into an rTMS-treated group (TMS group) and a control group.

Transcranial magnetic stimulation. High-frequency $(5 \mathrm{~Hz})$ rTMS was performed from post-TBI day 2 until the day before rats were sacrificed. A magnetic stimulator (BEMS-1, manufactured by the Institute of Electronic Engineering, Chinese Academy of Science, Beijing, China), a figure-of-eight coil and monophasic pulses were used (19). The coil had seven turns in each loop, with an inner radius of $3.5 \mathrm{~cm}$ and an outer radius of $5 \mathrm{~cm}$, which resulted in the whole rat brain being influenced by the induced magnetic field. Stimulation consisted of 36 trains of 25 pulses (each train lasted for $5 \mathrm{sec}$ ) with an inter-train interval of $15 \mathrm{sec}$ to prevent the coil from over-heating. A total of 900 pulses were given per day (20). The intensity was set at $60 \%$ of maximum output. The coil was placed over the head at a height of $\sim 0.5 \mathrm{~cm}$ and was not allowed to contact the scalp to rule out the influence of coil vibration. Careful attention was paid to ensure that maximal stimulation was over the center of the brain. Rats were awake during stimulation and secured in a small blanket. When rTMS was given to the TMS group, rats in the control group were placed in close proximity to the apparatus, so that they could hear the rhythmic sound produced by the coil.

Hematoxylin and eosin (HE) staining and calculation of relative brain parenchyma loss. At post-TBI days 7, 14 and 28, three or four rats from each group were deeply anesthetized by an intraperitoneal injection of chloral hydrate $(600 \mathrm{mg} / \mathrm{kg})$ and sacrificed by intracardial perfusion with chilled saline, until effluent was colorless, followed by $4 \%$ paraformaldehyde in phosphate-buffered saline (PBS; $20 \mathrm{~g}$ paraformaldehyde in $500 \mathrm{ml} \mathrm{PBS}$; pH 7.4). Brains were collected and fixed in $4 \%$ paraformaldehyde, then stored at $4^{\circ} \mathrm{C}$ overnight, before being transferred for paraffin embedding. A vibratome (Leica Microsystems GmbH, Wetzlar, Germany) was used to take a series of consecutive $3-\mu \mathrm{m}$ coronal sections from $1.5 \mathrm{~mm}$ behind the bregma (passing through the center of impact). Four sections from each rat were stained with HE, then photographed with a microscope using a x10 objective (Zeiss AG, Oberkochen, Germany). These sections were taken from a similar position in every rat, determined by the morphology of the lateral ventricle and striatum of the healthy hemisphere. The area of brain parenchyma in each section was evaluated using ImageJ v. 2.1.4.7 analysis software (National Institutes of Health, Bethesda, MA, USA). Relative brain parenchyma loss was then calculated as the percentage difference in parenchyma area between the impacted and non-impacted hemispheres.

Immunohistochemical (IHC) assessment. In order to detect endogenous cell proliferation, intraperitoneal injection of bromodeoxyuridine (BrdU; 50 mg/kg; Sigma-Aldrich; Merck KGaA, Darmstadt, Germany) was performed immediately after TBI, then every two days until sacrifice (21). Serial coronal sections were taken within $2 \mathrm{~mm}$ of the impacted point of the brain. Eight sections (3- $\mu \mathrm{m}$ thick) taken from similar positions in each rat (for example, from across the anterior part of the lateral ventricle) were used for IHC staining. Before immunostaining for BrdU, sections were first incubated in $50 \%$ formamide, $2 \mathrm{X}$ standard saline citrate for $2 \mathrm{~h}$ at $65^{\circ} \mathrm{C}$, followed by $2 \mathrm{~N}$ hydrochloric acid for $30 \mathrm{~min}$ at $37^{\circ} \mathrm{C}$. The sections were then blocked with $5 \%$ donkey serum (Beijing Dingguo Changsheng Biotechnology Co., Ltd., Beijing, China) and $0.3 \%$ Triton X-100 in PBS for $30 \mathrm{~min}$ 
at $37^{\circ} \mathrm{C}$. Sections were incubated at $4^{\circ} \mathrm{C}$ overnight with antibodies against BrdU [sheep polyclonal immunoglobulin (Ig)G; 1:200; ab1893; Abcam, Cambridge, UK], musashi1 [a marker for neural stem/progenitor cells (NSPCs); rabbit monoclonal IgG; 1:100; ab52865; Abcam], neuronal nuclei (NeuN; a marker for mature neurons; mouse monoclonal $\mathrm{IgG}$; 1:200; MAB377; EMD Millipore, Billerica, MA, USA) and caspase-3 (a marker for apoptosis; rabbit monoclonal IgG; 1:100; ab179517; Abcam). The specificities of these antibodies were accepted based on the manufacturers' technical information. Following this, sections were rinsed three times with PBS, for $5 \mathrm{~min}$ each, and incubated in the dark for $2 \mathrm{~h}$ at room temperature with fluorescein isothiocyanate-conjugated donkey anti-chicken (703-095-155), Cy5-conjugated donkey anti-rabbit (711-175-150) or Cy3-conjugated donkey anti-mouse secondary antibodies (711-165-151; all 1:500; all Jackson ImmunoResearch Laboratories, Inc., West Grove, PA, USA). Subsequently, the sections were rinsed three times, for 5 min each, with PBS. Following this, the sections were incubated at room temperature for $1 \mathrm{~h}$ with streptavidin-peroxidase using a VECTASTAIN ABC horseradish peroxidase kit (PK-4004; Vector Laboratories, Inc., Burlingame, CA, USA), rinsed three times with $\mathrm{PBS}$, for $5 \mathrm{~min}$ each, and visualized with $0.1 \mu \mathrm{g} / \mathrm{ml} \mathrm{4',6-diamidino-2-phenylindole} \mathrm{(D9542;}$ Sigma-Aldrich; Merck KGaA). The visualizing process was monitored under a microscope. Once sufficiently stained, after 5-10 $\mathrm{min}$, the sections were rinsed with PBS for $10 \mathrm{~min}$. To act as negative staining controls, sections from each rat received identical preparations for IHC staining, except that the primary antibodies were omitted. Sections were observed under a light microscope with an image acquisition system (Leica MC3000; Leica Microsystems GmbH, Wetzlar, Germany) and quantified using ImageJ v. 2.1.4.7 software. Staining of BrdU, NeuN and caspase-3 was assessed by the number of positive cells in a x200 field, while staining of musashil was evaluated by the percentage of musashil-positive area in total brain parenchyma area in a x200 field. For BrdU and musashil, fields with the highest positive cell count were selected from within the dorsolateral subventricular zone (SVZ) (22). Similar fields in the perilesional zone were selected for NeuN and caspase-3.

Micro-positron emission tomography (PET) examination. In order to assess glucose metabolic activity in the perilesional zone, a small number of rats ( $n=3$ per group) were subjected to fludeoxyglucose F $18\left({ }^{18} \mathrm{~F}-\mathrm{FDG}\right)$ micro-PET scanning at post-TBI days 2 and 13. ${ }^{18}$ F-FDG was produced with an RDS111 cyclotron and corresponding radiochemical synthesis system (both CTI Molecular Imaging; Siemens AG, Munich, Germany). Rats were anesthetized by intraperitoneal injection of chloral hydrate (400 mg/kg) and injected with $0.5 \mathrm{mCi}{ }^{18} \mathrm{~F}-\mathrm{FDG}$ dissolved in $0.4 \mathrm{ml}$ saline into the tail vein. Each animal was scanned with an Inveon dedicated micro-PET system (Siemens AG) following an uptake period of $20 \mathrm{~min}$. The system parameters were set as described by Constantinescu and Mukherjee (23). A 10-min static acquisition was performed in three-dimensional mode, and images were reconstructed using a maximum-a-posteriori probability algorithm. Images acquired from micro-PET were analyzed using ASIPro VM v. 6.0.5.0 software (Concorde Microsystems, Knoxville, TN, USA). A region of interest (ROI) method was used to evaluate change in the regional uptake of PET tracer. The relative metabolic activity was expressed as percentage deficit as compared with the contralateral region for ROIs (the cortex and striatum) in the perilesional zone, as described by Visnyei et al (24).

Statistical analysis. Data were analyzed using SPSS 19.0 software (IBM SPSS, Armonk, NY, USA). Data are presented as the mean \pm standard deviation. An independent-samples t-test was used to evaluate the heterogeneity of baseline data. The difference in unexpected mortality rate between the two groups was evaluated using Fisher's test. For each behavioral test, repeated-measures analysis of variance (ANOVA) was used to test for the inter-group variance as a result of the repeated assessments. The analysis consisted of testing for the group $\mathrm{x}$ time interaction. As no group $\mathrm{x}$ time interaction was detected, univariant ANOVA was used to access the differences between two groups at all time points. Independent-samples t-tests were also used to evaluate IHC results, relative brain parenchyma loss and relative metabolic activity. $\mathrm{P}<0.05$ was considered to indicate a statistically significant difference.

\section{Results}

Unexpected deaths of injured rats. After weight-dropping induced TBI, 33 rats survived until post-TBI day 2, when TMS commenced. A number of rats $(n=8)$ succumbed unexpectedly before the planned timings; however, the majority $(n=25)$ were sacrificed as planned. The mNSSs at post-TBI day 1 in unexpected mortality and planned sacrifice rats were similar $(10.00 \pm 2.03$ vs. $9.66 \pm 1.86, \mathrm{P}=0.568)$. The unexpected mortality rate in the TMS group $(8.33 \%, 1 / 12)$ was lower than that of the control group $(33.33 \%, 7 / 21)$, but this was not found to be a statistically significant result (Fig. 1).

Behavioral recovery after TBI. Rats with an mNSS of 9-14 and surviving for at least 14 days were evaluated for behavioral recovery. The number of rats with a score of 9-14 and surviving for 28 days was too small $(\mathrm{n} \leq 2)$ for statistical analysis, so these data are not shown. On post-TBI day 1, the mNSSs of the TMS group $(n=6)$ and control group $(n=5)$ were similar (Fig. 2; $10.17 \pm 1.17$ vs. $10.60 \pm 1.82, \mathrm{~F}=0.230, \mathrm{P}=0.643)$, as were the $\mathrm{mNSSs}$ of the two groups on day $7(7.00 \pm 1.27$ vs. $6.20 \pm 1.92$, $\mathrm{F}=0.689, \mathrm{P}=0.428$ ). The $\mathrm{mNSS}$ of the TMS group on post-TBI day 14 was lower than that of the control group, although this result was not found to be statistically significant (3.38 \pm 1.47 vs. $5.40 \pm 1.14, \mathrm{~F}=3.758, \mathrm{P}=0.09$ ).

Relative brain parenchyma loss in impacted hemisphere. Between post-TBI days 1-28, relative brain parenchyma loss in the impacted hemisphere increased in both groups. As shown in Fig. 3, differences between the TBI group and the control group at post-TBI day 7 and 14 were not significant. At post-TBI day 28 , relative brain parenchyma loss in the impacted hemisphere was observed to be lower in the TMS group than in the control group, although this difference was not found to be statistically significant. The typical view of the SVZ corner could not be observed as the brain tissue surrounding the dorsolateral ventricles of the injured hemispheres was necrotic. 


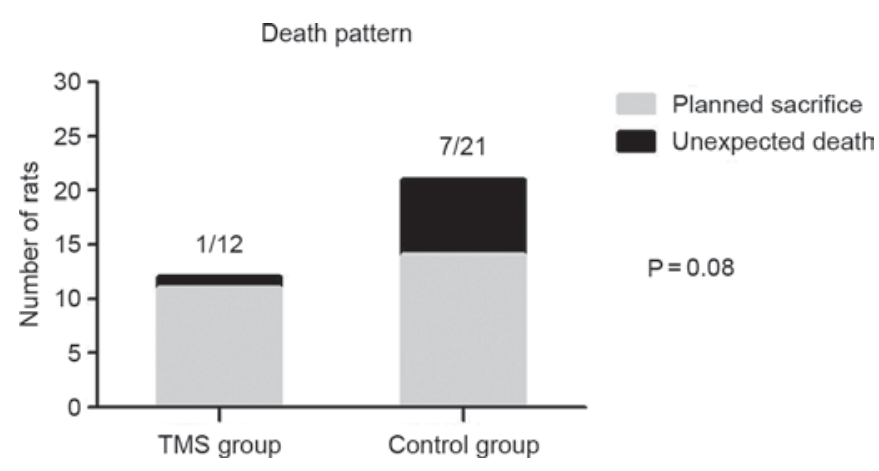

Figure 1. Unexpected death rate in rats after weight-dropping induced traumatic brain injury. TMS: Transcranial magnetic stimulation.

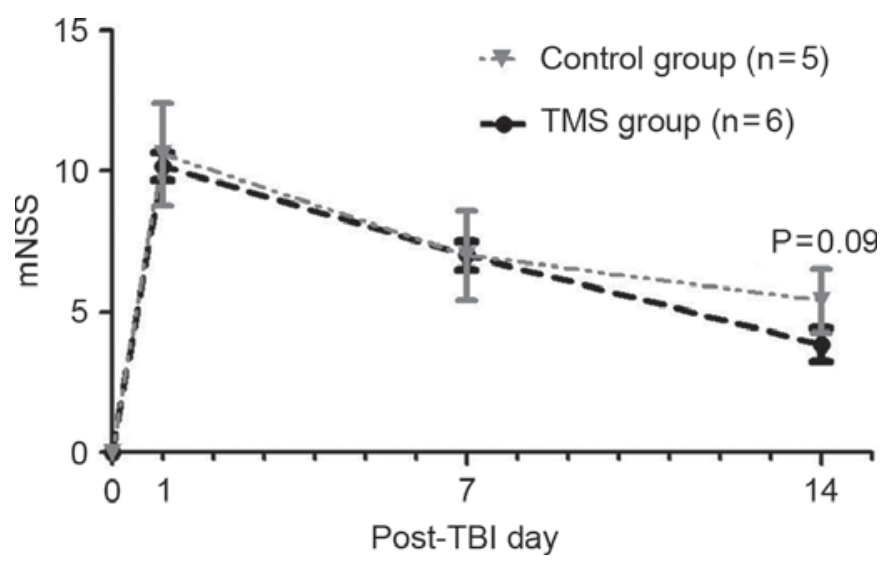

Figure 2. Behavioral recovery of TBI rats, as indicated by mNSS. TBI, traumatic brain injury; TMS, transcranial magnetic stimulation; mNSS, modified neurological severity score.
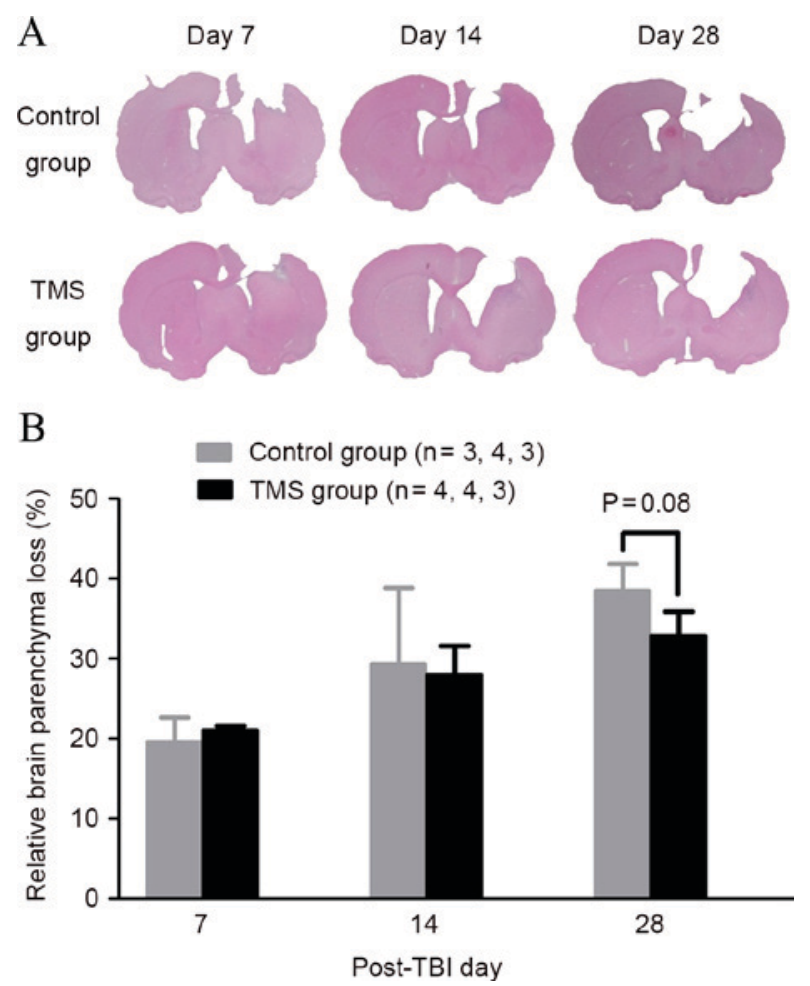

Figure 3. Relative brain parenchyma loss in the impacted hemispheres of TBI rats. (A) Example sections showing hematoxylin and eosin staining. (B) Relative brain parenchyma loss of TBI rats. TBI, traumatic brain injury; TMS, transcranial magnetic stimulation
Assessment of cell proliferation and neurogenesis in the dorsolateral SVZ. In the dorsolateral SVZ, BrdU positive cell density and musashi1 positive area (indicating cell proliferation and NSPCs, respectively) were highest on post-TBI day 14 in both groups (Fig. 4). The BrdU-positive cell density and musashil positive area were higher in the TMS group than the control group at day 7, 14 and 28. The BrdU-positive cell density was significantly higher in the TMS group compared with the control group at post-TBI day $14(175 \pm 22$ vs. $120 \pm 18, \mathrm{P}=0.02)$.

Assessment of neuron protection and cell apoptosis in the perilesional zone. The NeuN-positive cell densities (indicating mature neurons) in the perilesional zones were higher in the TMS group than in the control group at day 7, 14 and 28 (Fig. 5). At day 28, the NeuN-positive cell density was significantly higher in the TMS group compared with the control $(143 \pm 6$ vs. $106 \pm 8, \mathrm{P}=0.04$ ). Caspase- 3 positive cell density (indicating cell apoptosis) in the perilesional zone decreased from day 7 to day 28 in both groups. The caspase-3-positive cell density was significantly lower in the TMS group compared with the control group at post-TBI day $7(12 \pm 6$ vs. $31 \pm 5, \mathrm{P}=0.03)$ and day $14(8 \pm 4$ vs. $20 \pm 9, \mathrm{P}=0.01$ ).

Metabolic glucose activity in the perilesional zone. Compared with the same sites in the non-impacted hemisphere, the metabolic glucose activity in two ROIs (the perilesional zone in the cortex and the striatum of the impacted hemisphere) was reduced on post-TBI day 2 but elevated on post-TBI day 13 in both the TMS and control groups. No significant difference was observed between the two groups (Fig. 6).

\section{Discussion}

In the current study, the unexpected mortality rate in the TMS group was lower than in the control group, although this difference was not found to be statistically significant. The mNSSs of both groups at post-TBI day 1 were similar, so it is unlikely that the difference in unexpected mortality rate was caused by differences in TBI severity between the groups. Unexpected mortality was highest at post-TBI days 3-6, and the present observations suggest that the prominent cause of mortality after post-TBI day 2 was severe diarrhea, which was likely to have been induced by stress (25). It has previously been reported that rTMS can relieve stress (26-29), although these studies used inhibitory (low-frequency) rTMS. However, in the early phase of TBI pathophysiology, regional ischemia is the most important factor contributing to neural injury (10), and it is reported that excitatory (high-frequency) rTMS can increase regional blood flow (13). It is possible that rTMS caused a beneficial change in regional blood flow. Additionally, high-frequency rTMS can increase the level and enhance the pathway of $\operatorname{BDNF}(15,20)$, a neuroprotective factor. However, the difference between the groups was not statistically significant in the current study, so the effect of high-frequency rTMS on the survival of rats following moderate TBI has not been confirmed.

Another cause of unexpected mortality in the current study was epilepsy. Low-frequency rTMS has been reported to decrease the incidence of epilepsy (30). In the current study, no epileptiform seizures were observed in the TMS group, and seizures were only witnessed in two rats in the control 
A
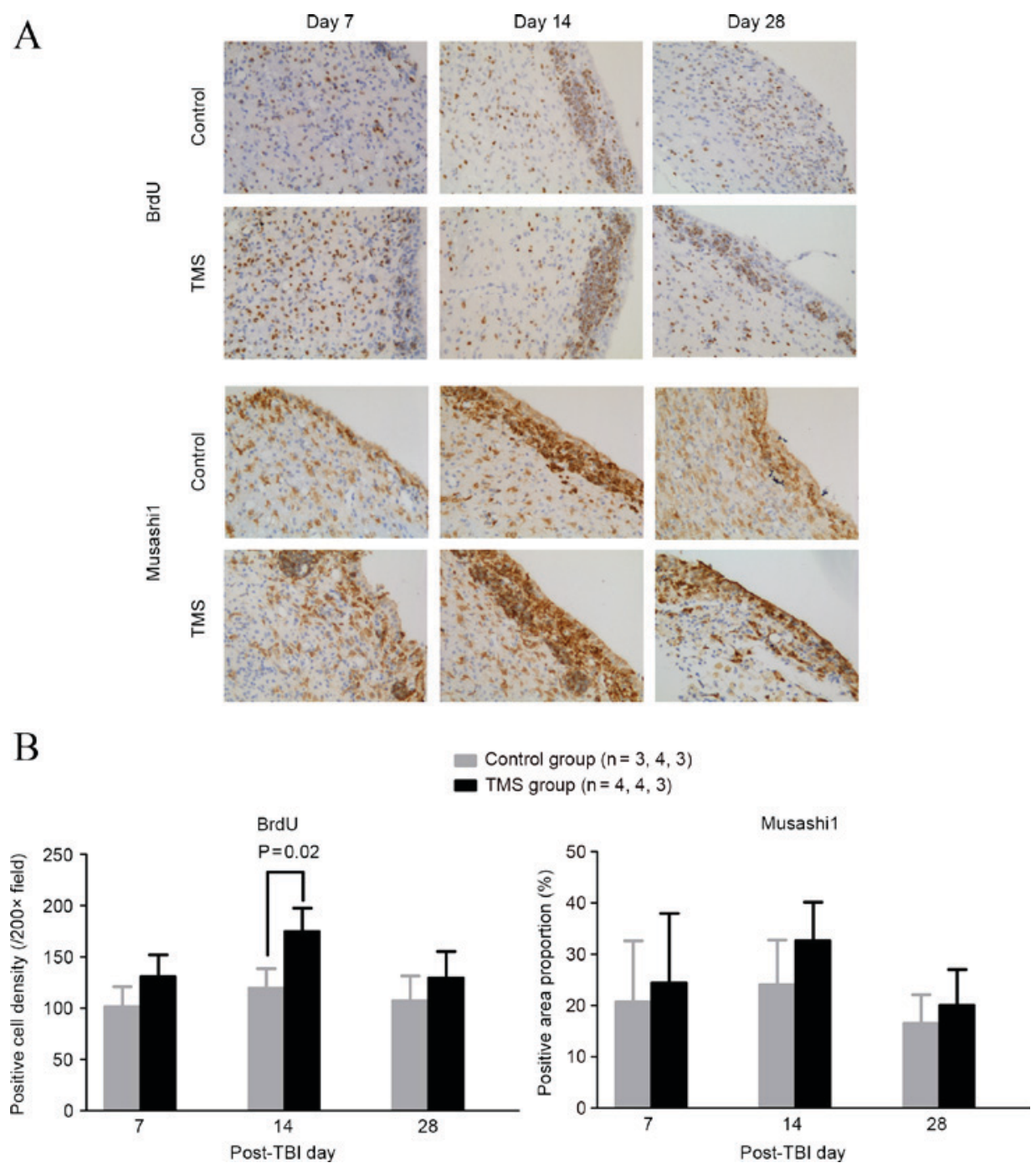

Figure 4. IHC analysis of cell proliferation and neurogenesis in the dorsolateral SVZ. (A) Example sections showing IHC staining for BrdU (to indicate proliferating cells) and musashil (to indicate neural stem/progenitor cells). Fields from the dorsolateral SVZ with the highest positive cell count were selected (magnification, x200). The typical view of the SVZ corner could not be observed because brain tissue surrounding the dorsolateral ventricles of the injured hemispheres was necrotic, as shown in Fig. 3. (B) Results of staining analysis. SVZ, subventricular zone; BrdU, bromodeoxyuridine; IHC, immunohistochemical; TBI, traumatic brain injury; TMS, transcranial magnetic stimulation.

group. Both of these animals passed away within $24 \mathrm{~h}$ of the seizure. Epilepsy has previously been reported as a complication of excitatory rTMS (7), although the incidence is low. It is unlikely that fatal epilepsy was a principal reason for the difference in unexpected mortality rate. However, it is possible that some seizures were not recorded, so a firm conclusion as to whether $5 \mathrm{~Hz}$ rTMS increased or decreased the incidence of epilepsy after TBI cannot be made.

A previous clinical trial (31) suggested that high-frequency rTMS could promote rehabilitation of stroke patients. The underlying mechanism is thought to be the promotion of neuroplasticity (7). During the late phase of recovery from TBI, residual neural networks may be reconstructed (5), perhaps partially compensating for the function of lost parenchyma. High-frequency rTMS might improve recovery of behavior after TBI by promoting adaptive plasticity (5), or repair of neural circuits (32). Yoon et al reported that rTMS did not have beneficial effects on motor recovery during the early stages of TBI in rats (33). The current results suggested that high-frequency rTMS may promote rehabilitation of rats after moderate TBI. However, these results were not found to be statistically significant. This is likely to be due to a small sample size, so it would be beneficial to repeat these experiments with a larger sample. According to the present observations, aside from improved mNSSs, rats subjected to rTMS also gained weight faster. This is a parameter that could be investigated further in future studies.

In the current study, the relative brain parenchyma loss in coronary brain sections following TBI was evaluated. The average values in the rTMS and control groups were similar at post-TBI day 7 and 14 . At day 28 , the relative brain parenchyma loss in the impacted hemisphere was lower in the TMS group than in the control group, although this result was not found to be statistically significant. When the gross brains were observed, a lower level of atrophy was observed in the impacted hemispheres of the TMS group compared with the control group. Again, this is a parameter that could be investigated in future studies.

Neuroprotective effects of high-frequency rTMS were observed in the results of IHC staining. The density of 
A
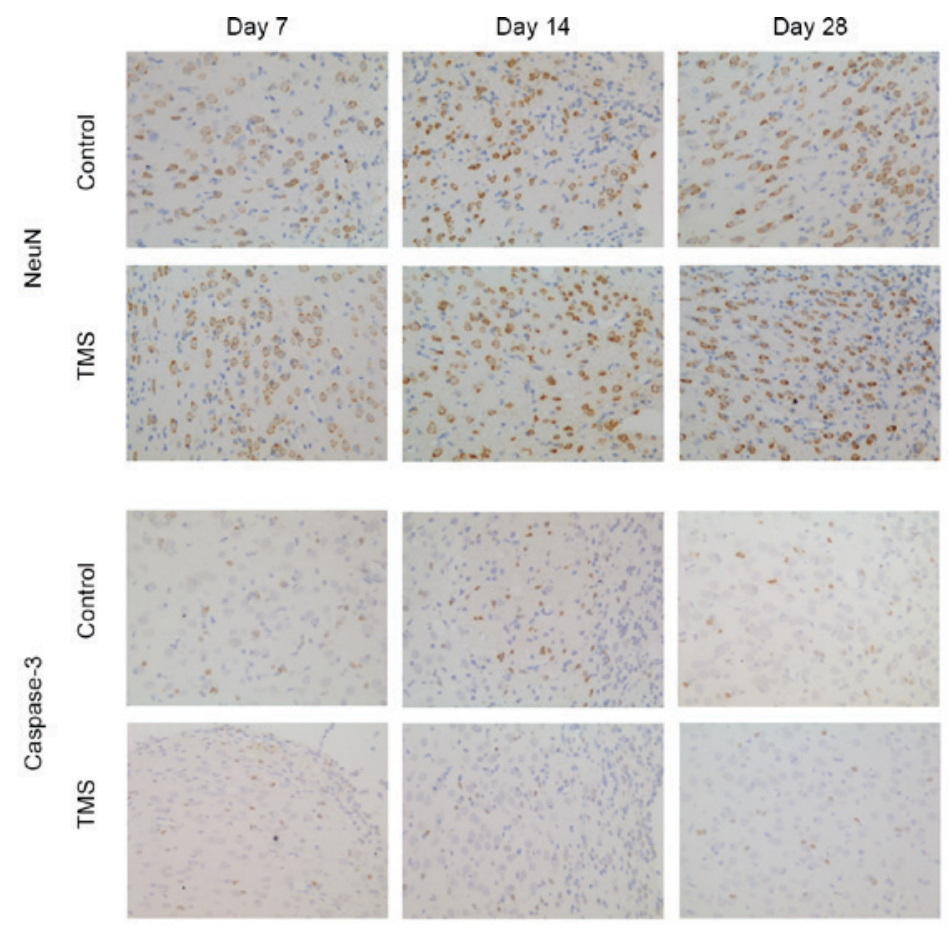

$\mathrm{B}$

Control group $(n=3,4,3$

- TMS group $(n=4,4,3)$
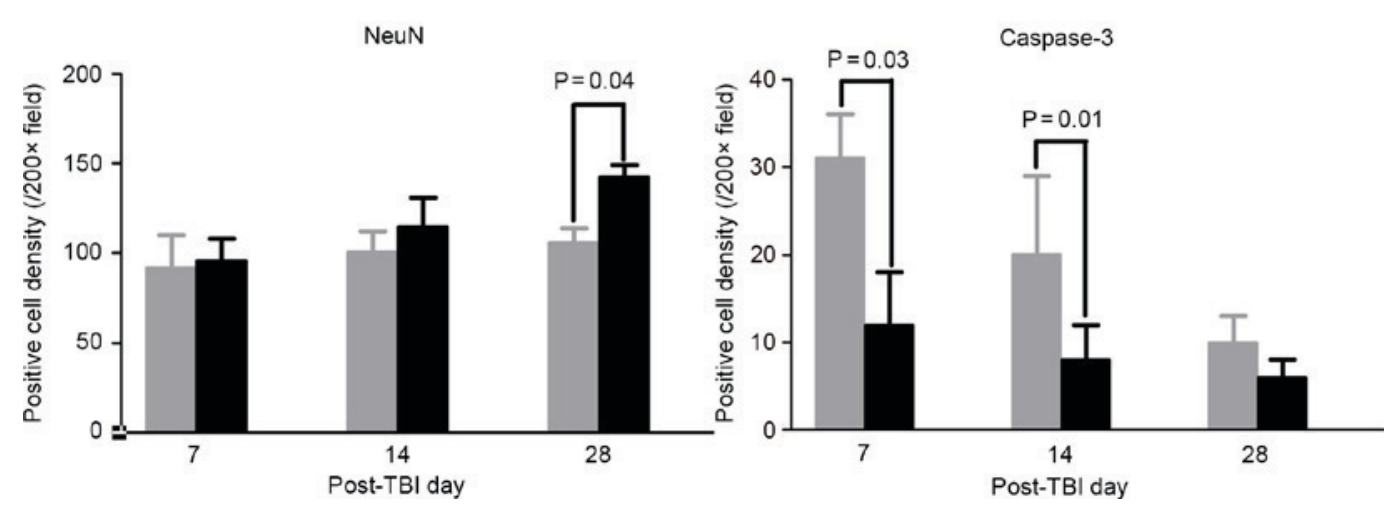

Figure 5. IHC analysis of neuron protection and cell apoptosis in the perilesional zone. (A) Example sections showing IHC staining of NeuN (indicating mature neurons) and caspase-3 (indicating cell apoptosis). Fields from the perilesional zone with the highest positive cell count were selected (magnification, x200). (B) Results of staining analysis. IHC, immunohistochemical; TBI, traumatic brain injury; TMS, transcranial magnetic stimulation.

perilesional cells positive for caspase-3, a well-known apoptosis marker, was significantly lower in the TMS group at post-TBI day 7 and 14 as compared with the control group. This suggested that TMS caused inhibition of apoptosis in the perilesional zone. Yoon et al also observed an anti-apoptosis effect of high-frequency $(10 \mathrm{~Hz}) \mathrm{rTMS}$ in the perilesional area (33). The density of cells positive for NeuN, a marker of mature neurons, was significantly higher in the TMS group compared with the control group at post-TBI day 28 , suggesting inhibition of mature neuron loss in the perilesional zone.

High-frequency rTMS can increase the level of BDNF and enhance functioning of the BDNF pathway. BDNF is a neurogenesis stimulator (22). It may therefore be the case that high-frequency rTMS can promote neurogenesis, in much the same way as other NBS methods (34-36). In a previous study, in rats that suffered middle cerebral artery occlusion, cell proliferation (indicated by $\mathrm{BrdU}$ ) was highest around 14 days after injury (37). This is consistent with the results of the current study; BrdU positive cell density in the SVZ at post-TBI day 14 was significantly higher in the TMS group compared with the control group. However, increased cell proliferation does not necessarily equate to increased neurogenesis because newly created inflammatory cells are also BrdU positive. For this reason, the tissues were also stained for musashi1, a marker of NSPCs. The results did show musashil to be significantly higher in the TMS group than the control group. The level of neurogenesis can also be reflected by metabolic changes; however, in the current study, no significant differences in glucose metabolic activity in the perilesional zone were observed between the TMS and control groups.

In conclusion, the current study observed a trend indicating that high-frequency rTMS may decrease mortality and improve behavioral recovery in rats suffering from moderate TBI. This suggests that high-frequency rTMS has potential as a neuroprotective treatment for TBI. These findings were 
A

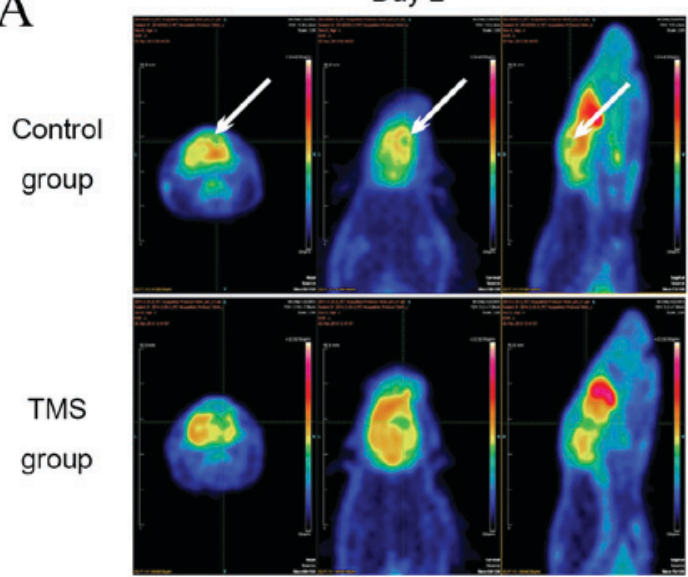

Day 13

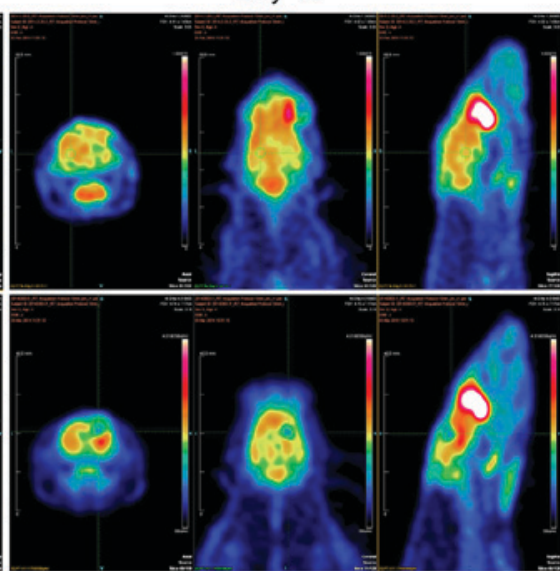

B

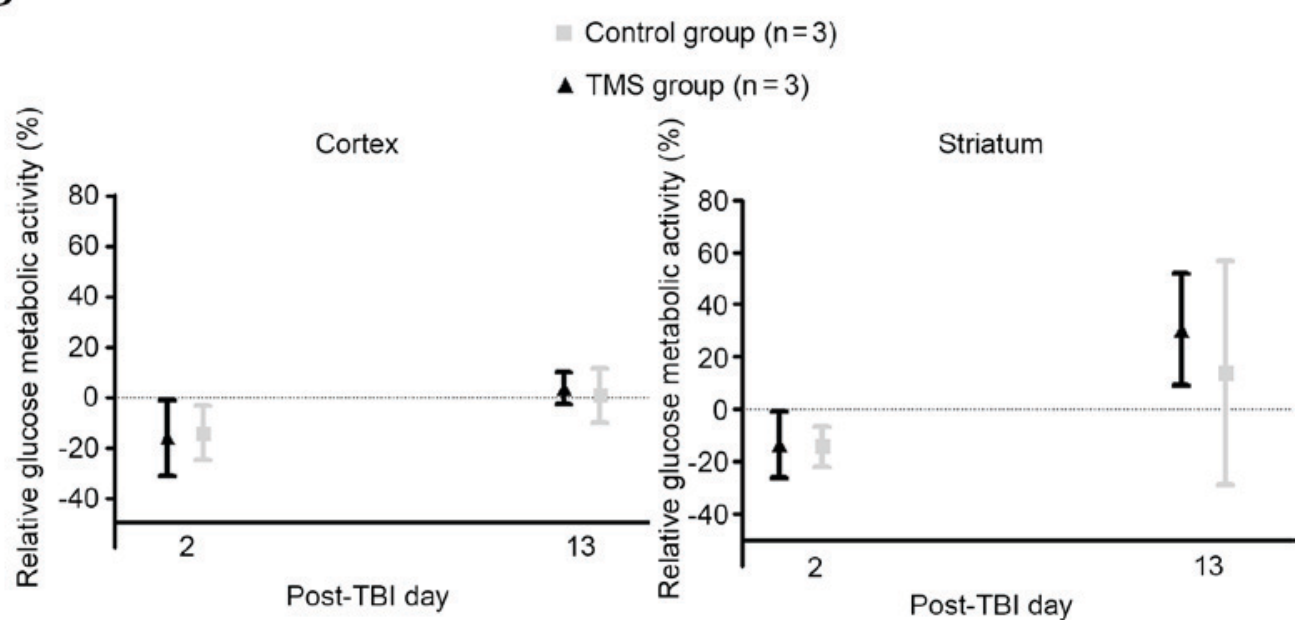

Figure 6. Metabolic glucose activity in the perilesional zone. (A) Example imaging from micro-positron emission tomography of TBI rats. The colors indicate the level of fludeoxyglucose F 18 uptake, with blue indicating a low level and white indicating a high level (see the color gradients on the right of each scan). Lesions induced by trauma are indicated with white arrows. Metabolic glucose activity of the impacted hemisphere was evaluated in two sites: The perilesional zone in the cortex, and the striatum. (B) Relative metabolic activity, as compared with the same site in the intact hemisphere. TBI, traumatic brain injury; TMS, transcranial magnetic stimulation.

supported by analysis at the cellular level; high-frequency rTMS appeared to promote cell proliferation and neurogenesis in the dorsolateral SVZ, as well as decrease mature neuron loss and reduce apoptosis in the perilesional zone. Glucose metabolic activity in the perilesional zone may decrease in the early phase following TBI, and then increase in the later phase, but this is not influenced by high-frequency rTMS. This was a pilot study and was thus limited by the small sample size. In order to reach definitive conclusions, experiments with a larger sample size and improved design are needed. To our knowledge, this is one of very few studies to have investigated the effect of rTMS on TBI. These findings may provide a reference for future investigations into the treatment of TBI with rTMS.

\section{Acknowledgements}

This research was supported by the National High Technology Research and Development Program of China (2014AA020513), the National Basic Research Program of China (grant no. 2014CB541603), and CAMS Initiative for Innovative Medicine (CAMS-12M) (2016-12M-017).

\section{References}

1. Algattas $\mathrm{H}$ and Huang JH: Traumatic brain injury pathophysiology, and treatments: Early, intermediate, and late phases post-injury. Int J Mol Sci 15: 309-341, 2014.

2. Faul M, Xu L, Wald MM and Coronado VG (eds): Traumatic Brain Injury in the United States: Emergency Department Visits, Hospitalizations, and Deaths. Centers for Disease Control and Prevention, National Center for Injury Prevention and Control, Atlanta, GA, USA, 2010.

3. Sosin DM, Sacks JJ and Smith SM: Head injury-associated deaths in the United States from 1979 to 1986. JAMA 262: 2251-2255, 1989.

4. Wu X, Hu J, Zhuo L, Fu C, Hui G, Wang Y, Yang W, Teng L, Lu S and $\mathrm{Xu}$ G: Epidemiology of traumatic brain injury in eastern China, 2004: A prospective large case study. J Trauma 64: 1313-1319, 2008.

5. Villamar MF, Santos Portilla A, Fregni F and Zafonte R: Noninvasive brain stimulation to modulate neuroplasticity in traumatic brain injury. Neuromodulation 15: 326-338, 2012.

6. Demirtas-Tatlidede A, Vahabzadeh-Hagh AM, Bernabeu M, Tormos JM and Pascual-Leone A: Noninvasive brain stimulation in traumatic brain injury. J Head Trauma Rehabil 27: 274-292, 2012.

7. Dayan E, Censor N, Buch ER, Sandrini M and Cohen LG: Noninvasive brain stimulation: From physiology to network dynamics and back. Nat Neurosci 16: 838-844, 2013.

8. Barker AT, Jalinous R and Freeston IL: Non-invasive magnetic stimulation of human motor cortex. Lancet 1: 1106-1107, 1985. 
9. Chen R, Classen J, Gerloff C, Celnik P, Wassermann EM, Hallett $M$ and Cohen LG: Depression of motor cortex excitability by low-frequency transcranial magnetic stimulation. Neurology 48: 1398-1403, 1997.

10. Pascual-Leone A, Valls-Solé J, Wassermann EM and Hallett M: Responses to rapid-rate transcranial magnetic stimulation of the human motor cortex. Brain 117: 847-858, 1994.

11. Orosz A, Jann K, Wirth M, Wiest R, Dierks T and Federspiel A: Theta burst TMS increases cerebral blood flow in the primary motor cortex during motor performance as assessed by arterial spin labeling (ASL). Neuroimage 61: 599-605, 2012.

12. Cho SS, Pellecchia G, Ko JH, Ray N, Obeso I, Houle S and Strafella AP: Effect of continuous theta burst stimulation of the right dorsolateral prefrontal cortex on cerebral blood flow changes during decision making. Brain Stimul 5: 116-123, 2012.

13. Kito S, Fujita K and Koga Y: Regional cerebral blood flow changes after low-frequency transcranial magnetic stimulation of the right dorsolateral prefrontal cortex in treatment-resistant depression. Neuropsychobiology 58: 29-36, 2008.

14. Speer AM, Kimbrell TA, Wassermann EM, D Repella J, Willis MW, Herscovitch P and Post RM: Opposite effects of high and low frequency rTMS on regional brain activity in depressed patients. Biol Psychiatry 48: 1133-1141, 2000.

15. Gersner R, Kravetz E, Feil J, Pell G and Zangen A: Long-term effects of repetitive transcranial magnetic stimulation on markers for neuroplasticity: Differential outcomes in anesthetized and awake animals. J Neurosci 31: 7521-7526, 2011.

16. Feeney DM, Boyeson MG, Linn RT, Murray HM and Dail WG: Responses to cortical injury: I. Methodology and local effects of contusions in the rat. Brain Res 211: 67-77, 1981.

17. Gao Y, Sun JZ, Tian ZX and Wu GY: An improved weight-dropping model of traumatic brain injury in rats. Zhe Jiang Chuang Shang Wai Ke Bian Ji Bu 9: 283-285, 2004 (In Chinese).

18. Schallert T, Kozlowski DA, Humm JL and Cocke RR Use-dependent structural events in recovery of function. Adv Neurol 73: 229-238, 1997.

19. Yue L, Xiao-lin H and Tao S: The effects of chronic repetitive transcranial magnetic stimulation on glutamate and gamma-aminobutyric acid in rat brain. Brain Res 1260 94-99, 2009

20. Wang HY, Crupi D, Liu J, Stucky A, Cruciata G, Di Rocco A, Friedman E, Quartarone A and Ghilardi MF: Repetitive transcranial magnetic stimulation enhances BDNF-TrkB signaling in both brain and lymphocyte. J Neurosci 31: 11044-11054, 2011.

21. Taguchi A, Soma T, Tanaka H, Kanda T, Nishimura $H$, Yoshikawa H, Tsukamoto Y, Iso H, Fujimori Y, Stern DM, et al: Administration of $\mathrm{CD}_{3} 4^{+}$cells after stroke enhances neurogenesis via angiogenesis in a mouse model. J Clin Invest 114: 330-338, 2004.

22. Guan J, Tong W, Ding W, Du S, Xiao Z, Han Q, Zhu Z, Bao X, Shi $\mathrm{X}, \mathrm{Wu} \mathrm{C}$, et al: Neuronal regeneration and protection by collagen-binding BDNF in the rat middle cerebral artery occlusion model. Biomaterials 33: 1386-1395, 2012.

23. Constantinescu CC and Mukherjee J: Performance evaluation of an Inveon PET preclinical scanner. Phys Med Biol 54: 2885-2899, 2009.
24. Visnyei K, Tatsukawa KJ, Erickson RI, Simonian S, Oknaian N, Carmichael ST and Kornblum HI: Neural progenitor implantation restores metabolic deficits in the brain following striatal quinolinic acid lesion. Exp Neurol 197: 465-474, 2006.

25. Hirata T, Keto Y, Nakata M, Takeuchi A, Funatsu T, Akuzawa S, Sasamata M and Miyata K: Effects of serotonin 5-HT3 receptor antagonists on stress-induced colonic hyperalgesia and diarrhoea in rats: A comparative study with opioid receptor agonists, a muscarinic receptor antagonist and a synthetic polymer. Neurogastroenterol Motil 20: 557-565, 2008.

26. Túnez I, Montilla P, del Carmen Muñoz M, Medina FJ and Drucker-Colín R: Effect of transcranial magnetic stimulation on oxidative stress induced by 3-nitropropionic acid in cortical synaptosomes. Neurosci Res 56: 91-95, 2006.

27. Tasset I, Drucker-Colin R, Peña J, Jimena I, Montilla P, Medina FJ and Túnez I: Antioxidant-like effects and protective action of transcranial magnetic stimulation in depression caused by olfactory bulbectomy. Neurochem Res 35: 1182-1187, 2010.

28. Tasset I, Pérez-Herrera A, Medina FJ, Arias-Carrión O, Drucker-Colín R and Túnez I: Extremely low-frequency electromagnetic fields activate the antioxidant pathway Nrf2 in a Huntington's disease-like rat model. Brain Stimul 6: 84-86, 2013.

29. Teyssier JR, Trojak B, Chauvet-Gelinier JC and Bonin B: Low frequency transcranial magnetic stimulation downregulates expression of stress genes in blood leucocytes: Preliminary evidence. J Psychiatr Res 47: 935-936, 2013.

30. Sun W, Fu W, Mao W, Wang D and Wang Y: Low-frequency repetitive transcranial magnetic stimulation for the treatment of refractory partial epilepsy. Clin EEG Neurosci 42: 40-44, 2011.

31. Sung WH, Wang CP, Chou CL, Chen YC, Chang YC and Tsai PY: Efficacy of coupling inhibitory and facilitatory repetitive transcranial magnetic stimulation to enhance motor recovery in hemiplegic stroke patients. Stroke 44: 1375-1382, 2013.

32. Rodger J and Sherrard RM: Optimising repetitive transcranial magnetic stimulation for neural circuit repair following traumatic brain injury. Neural Regen Res 10: 357-359, 2015.

33. Yoon YS, Yu KP, Kim H, Kim HI, Kwak SH and Kim BO: The effect of electric cortical stimulation after focal traumatic brain injury in rats. Ann Rehabil Med 36: 596-608, 2012.

34. Cuccurazzu B, Leone L, Podda MV, Piacentini R, Riccardi E, Ripoli C, Azzena GB and Grassi C: Exposure to extremely low-frequency $(50 \mathrm{~Hz})$ electromagnetic fields enhances adult hippocampal neurogenesis in C57BL/6 mice. Exp Neurol 226: $173-182,2010$.

35. Arias-Carrión O, Verdugo-Díaz L, Feria-Velasco A Millán-Aldaco D, Gutiérrez AA, Hernández-Cruz A and Drucker-Colín R: Neurogenesis in the subventricular zone following transcranial magnetic field stimulation and nigrostriatal lesions. J Neurosci Res 78: 16-28, 2004.

36. Rueger MA, Keuters MH, Walberer M, Braun R, Klein R, Sparing R, Fink GR, Graf R and Schroeter M: Multi-session transcranial direct current stimulation (tDCS) elicits inflammatory and regenerative processes in the rat brain. PLoS One 7: e43776, 2012

37. Bao X, Feng M, Wei J, Han Q, Zhao H, Li G, Zhu Z, Xing H, An Y, Qin C, et al: Transplantation of Flk- $1^{+}$human bone marrow-derived mesenchymal stem cells promotes angiogenesis and neurogenesis after cerebral ischemia in rats. Eur J Neurosci 34: 87-98, 2011. 